\title{
Meningioma Development Post Burr Hole Craniostomy
}

\section{Ling Jyh Chyang*, Mohamad Hidir Abdullah, Mohd Syahiran bin Mohd Sidek and Mohamad Azhari Omar}

\author{
Department of Neurosurgery, Hospital Raja Permaisuri Bainun Ipoh, Malaysia
}

*Corresponding author: Ling Jyh Chyang, Department of Neurosurgery, Hospital Raja Permaisuri Bainun Ipoh, Jalan Raja Ashman Shah, 30450 Ipoh, Perak, Malaysia, Tel: +605-2085000

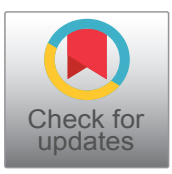

\begin{abstract}
Meningiomas are the most common primary intracranial tumors. They arise from meningothelial cells of the arachnoid layer. Trauma as a cause for meningioma has been mentioned in literatures but still remains controversial. We present a case of 69-year-old lady who developed a large meningioma at previous site of burrhole-craniostomy within 6 months period.
\end{abstract}

\section{Introduction}

Meningiomas are the most common primary intracranial tumors. They arise from meningothelial cells of the arachnoid layer. Common risk factors associated with meningiomas are ionizing radiation and neurofibromatosis. Trauma as a cause for meningioma has been mentioned in literatures but still remains controversial. To our knowledge, there aren't any reports on development of meningioma at a previous operative site, hence we present a case of meningioma development at the site of previous burrhole craniostomy. The tumor grew to a significant size of $4 \mathrm{~cm}$ diameter within the period of 6 months, but histopathological examination showed a benign nature WHO Grade I tumor.

\section{Case Presentation}

A 69-year-old lady with no known comorbids, presented to us with 2 weeks history of incoherent speech, and altered behaviour. An MRI Brain showed a solitary homogenously enhancing extra-axial broad based lesion over the left temporal convexity, which measures $4.5 \times 3 \mathrm{~cm}$.

Further history revealed that she has had an operation done 6 months prior for left convexity chronic subdural hematoma. CT Brain and MRI Brain scans (Figure 1) showed chronic subdural hematoma of 1.5 $\mathrm{cm}$ thickness. She underwent left burr-hole craniostomy and drainage of hematoma with placement of a subdural drain. Both pre-operative CT and MRI scans showed no evidence of tumor presence at that time.

A craniotomy for tumor excision was done during this admission, which we achieved a Simpson Grade 1 excision. Histopathological examination confirmed meningothelial meningioma (WHO Grade I). Followup post-operative MRI Brain at 6 months showed no evidence local tumor recurrence.

\section{Discussion}

Meningioma is the most common primary intracranial tumor in adults, accounting for about a third of all primary CNS neoplasms. Meningiomas originate from meningothelial arachnoid cap cells, they occur primarily at the base of the skull in the parasellar regions and over the cerebral convexities [1].

Both environmental and genetic factors have been implicated in meningioma formation, although most examples are idiopathic. These neoplasms are more common during the middle decades of life, incidence rates increase with age, higher in adults age 65 and older. There is a female predominance with female-male ratio of approximately 2:1. World Health Organization (WHO) classify meningiomas into three grades, benign (Grade I, 90\%), atypical (Grade II, 5\%), anaplastic (Grade III, 3-5\%) [2], in which grade I meningiomas are more common in women, whereas higher-grade meningiomas have a slight male predominance.

Proposed environmental factors include irradiation, 


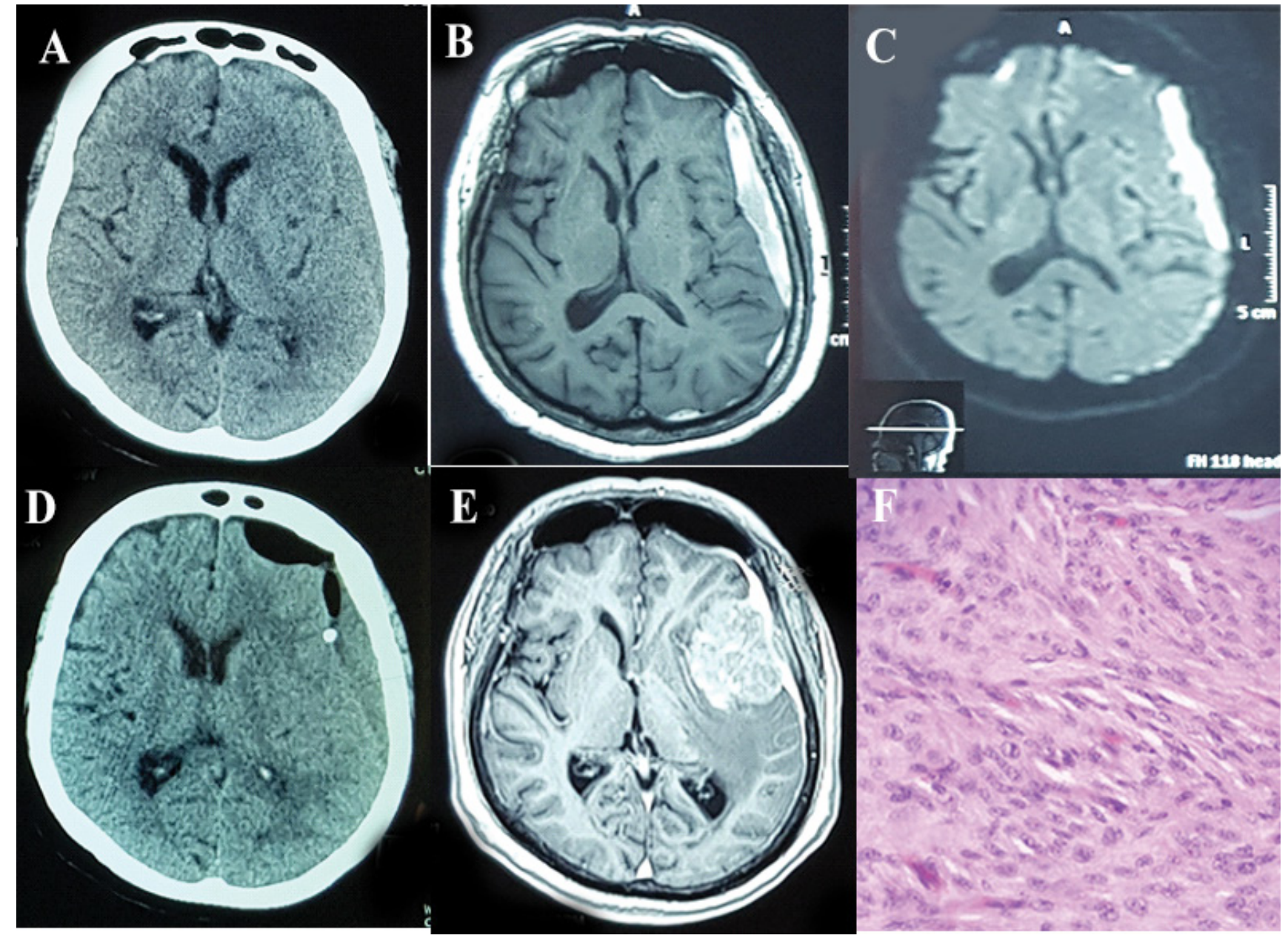

Figure 1: A) Pre-operative CT brain and MRI brain; B) Post gadolinium; C) DWI showing left convexity chronic SDH, but did not show any evidence of extra-axial tumor; D) Post burr-hole craniostomy CT Brain, with subdural drain seen in subdural space; E) MRI Brain 6 months later after burr-hole showing large left temporal broad based tumor; F) HPE slide confirmed Meningothelial meningioma.

hormone exposures, and head trauma, have been associated with development of meningioma. In Cushing and Eisenhardt's 1938 monograph, a surprising 32\% of intracranial meningiomas had a prior history of head injury, and a modern epidemiologic study estimated a 4.33 odds ratio for developing meningiomas more than 10 years after head trauma [3].

As far as histopathogenesis is concerned, a trauma of sufficient entity may facilitate the development of a meningioma, particularly when there is an accompanying meningeal lesion with the implantation of foreign bodies or a granulomatous reaction. In such circumstances, it is possible that the meningeal tissue undergoes aneoplastic change during the reparative processes.

Barnett, et al. have suggested that a correlation exists between chronic inflammation leading to meningeal irritation, cellular atypia of the meningeal tissue, facilitating neoplastic growth [4].

As established by Ewing and Zulch, the diagnosis of post-traumatic meningiomas requires the simultaneous existence of the specific features such as: 1) The site of the head injury and meningioma is the same; 2) The trauma was authentic and of sufficient entity; 3) The previous integrity of the region before the trauma is ascertained; 4) The time interval between the two events is significant; 5) There is continuity in the pathological changes; 6) The tumour is verified histologically. All of these diagnostic criteria were present in our patient [5].

In Grade 1 meningiomas, the mean absolute growth rate was $1.51 \mathrm{~cm}^{3} /$ year, the median relative growth rate and tumor doubling time was $14.18 \%$ /year and 5.228 years. Higher grade meningiomas have higher absolute and relative growth rates and shorter tumor doubling time [6].

In most publications, post-traumatic meningiomas are described as atypical or malignant. In our case, the patient had a benign type of meningioma but with an above average growth rate.

\section{Conclusion}

As in our case, an injury to the arachnoid cells during burr-hole craniostomy and insertion of a subdural drain, likely led to over proliferation of the arachnoid cells and neoplastic meningeal tissue transformation during the course of wound healing process. Patients with post- 
traumatic meningioma may have benign nature of tumor but with higher tumor growth rate than average.

\section{Funding}

No funding was received that could have influenced the outcome.

\section{Author's Contribution}

LJC, MH, MS, and MA are involved in planning, and development of study concept. MS and MA are involved with the surgery. $\mathrm{LC}, \mathrm{MH}, \mathrm{MS}$ and MA contributed to the writing of the manuscript.

\section{References}

1. Kilani M, Darmoul M, Hammedi F, Ben Nsir A, Hattab MN (2015) Cavernous hemangioma of the skull and meningioma: Association or coincidence?. Case Rep Neurol Med 2015: 716837.
2. Claus EB, Bondy ML, Schildkraut JM, Wiemels JL, Wrensch $\mathrm{M}$, et al. (2005) Epidemiology of intracranial meningioma. Neurosurgery 57: 1088-1095.

3. Arie Perry (2017) Practical surgical neuropathology. (2 $2^{\text {nd }}$ edn), Elsevier Health Sciences.

4. Barnett GH, Chou SM, Bay JW (1986) Posttraumatic intracranial meningioma: A case report and review of the literature. Neurosurgery 18: 75-78.

5. Cervoni L, Celli P, Maraglino C, Gagliardi FM (1996) Intracranial meningioma at the site of a previous cranial fracture: Case report and review of the literature. Ital J Neuro Sci 17: 79-81.

6. Nakamura M, Roser F, Michel J, Jacobs C, Samii M (2005) Volumetric analysis of the growth rate of incompletely resected intracranial meningiomas. Zentralbl Neurochir 66: 17-23. 